\title{
The accuracy of lung ultrasonography to identify the position of left sided double lumen tracheal tube in elective thoracic surgery: a preliminary study.
}

Tae-Jun Kim, Hyungseok Seo, Bong Jae Lee, and Dong-Ok Kim

Department of Anesthesiology and Pain Medicine,

Kyung Hee University Hospital at Gangdong,

Kyung Hee University College of Medicine, Seoul, Korea

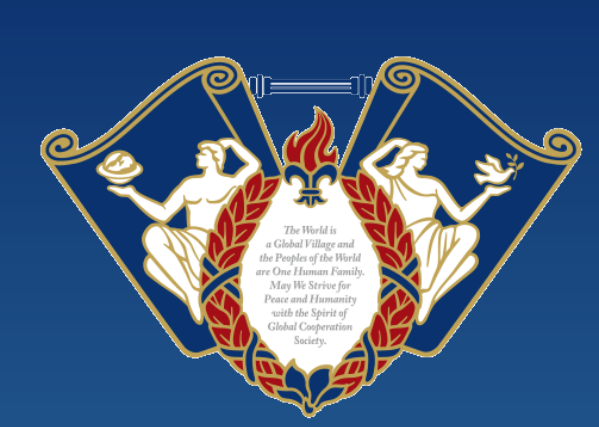

\section{INTRODUCTION}

\section{One lung ventilation in thoracic surgery}

- In thoracic surgery, a double lumen tracheal tube is required for one-lung

ventilation.

- Rapid identification of the accurate position of the double lumen tube is

important.

- Fiberoptic bronchoscopy is confirmative technique, but it requires specific device and experienced staff.

Identification of double lumen tracheal tube position - Chest auscultation

easy, rapid technique, but individual and less sensitive

- hypoventilation can be identified by decreased breath sound

- Lung sonography

require sonography, but relatively easy, visualizing, more informative

hypoventilation can be identified by decrease or no pleural movement

\section{OBJECTIVES}

We compared the accuracy of two methods (chest auscultation and lung sonography) for the detection of appropriate position of left sided double lumen tracheal tube.

\section{METHODS}

$>$ This is a prospective observation study

$>$ Inclusion criteria

(1) $>19$ years old adults

(2) ASA PS 1 or 2

$>$ Exclusion criteria

(1) $\mathrm{BMI}<18.5$ or $>35 \mathrm{~kg} / \mathrm{m}^{2}$

(2) Patients with severe chest deformity

(3) Patients with history of tracheostomy

(4) Patients with anticipated of difficult intubation

(5) Patients with history of lung resection

(6) Patients with history of pleural disease or pleura surgery

$>$ Detection of DLT tube position using lung sliding sign
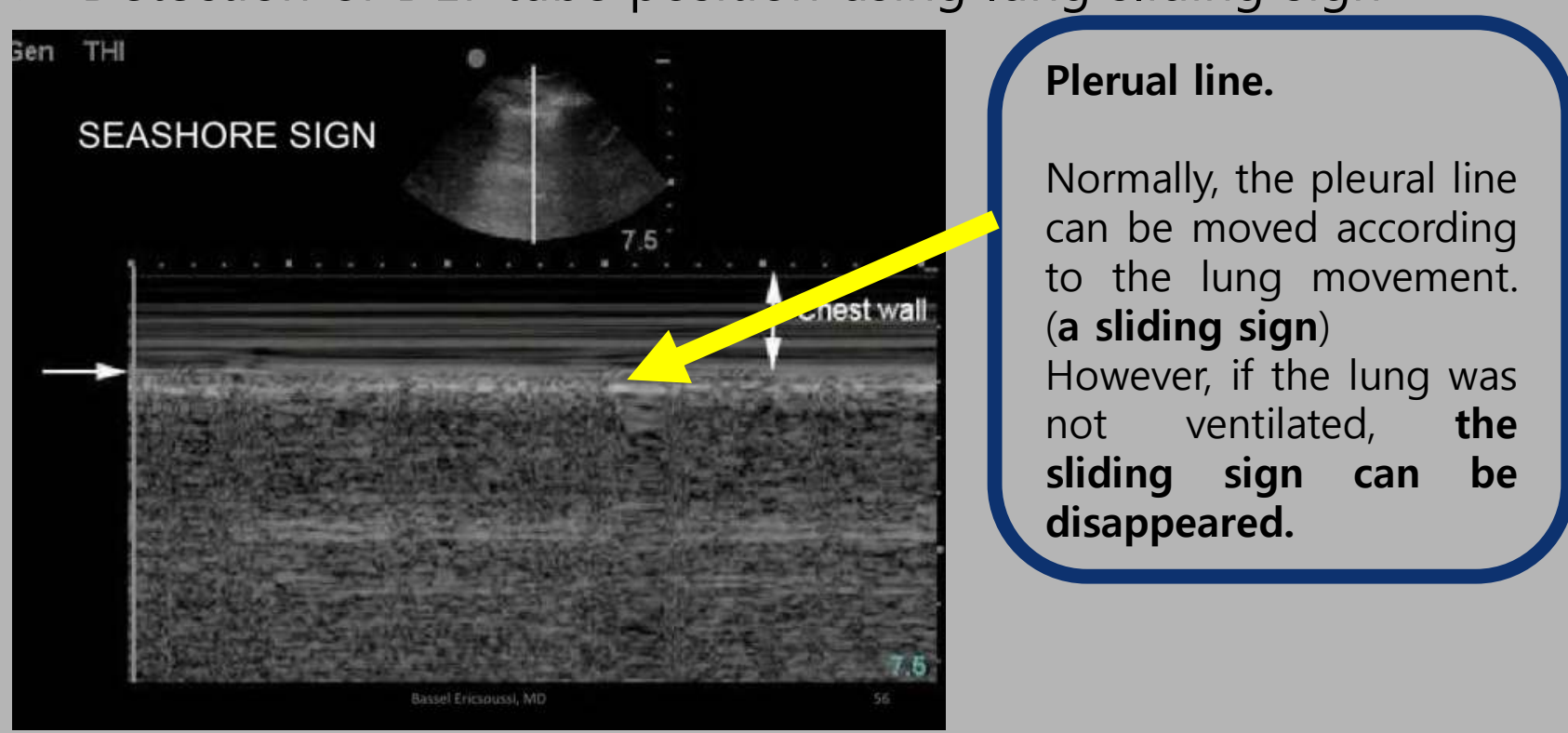

$>$ Primary outcome

Accuracy of each procedure using $2 \times 2$ table$$
\text { FOB }
$$

\begin{tabular}{c|c|c|} 
Aus/Sono & Correct & Incorrect \\
\hline
\end{tabular}

correct

\begin{tabular}{|l|l|}
\hline$A$ & $B$ \\
\hline$C$ & $D$ \\
\hline
\end{tabular}

Accuracy $=$

$\frac{A+D}{A+B+C+D}$

\section{STUDY PROTOCOL}

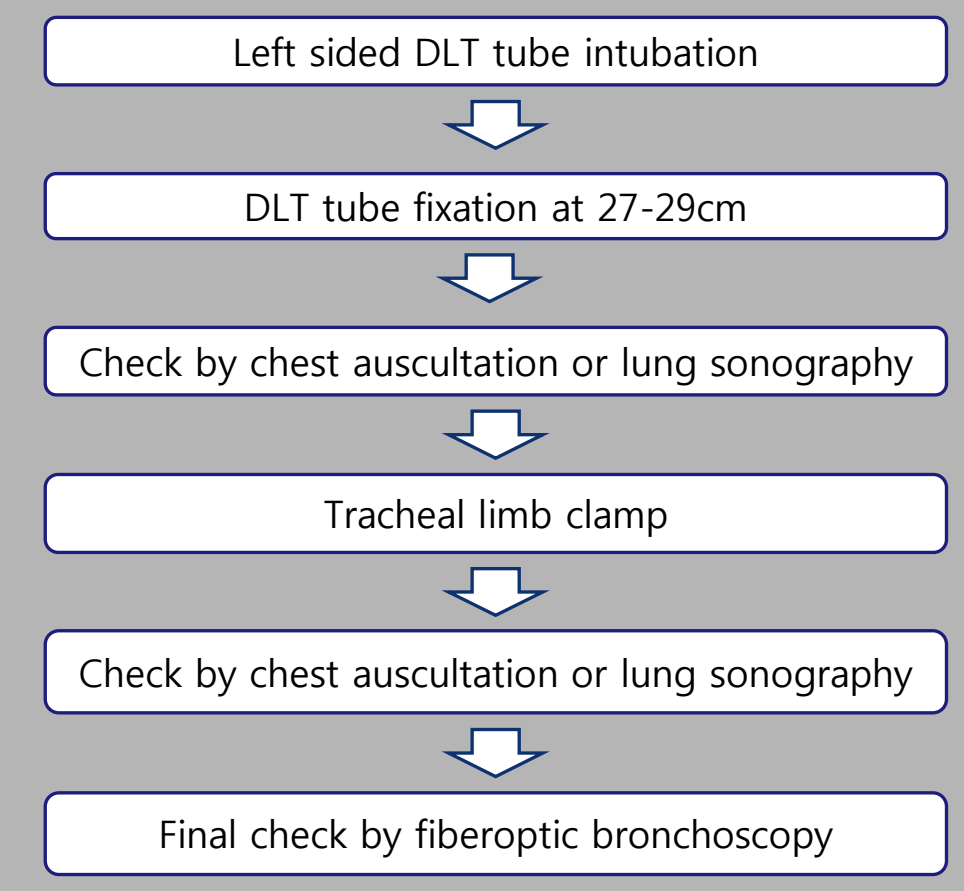

\section{RESULTS}

Demographics and intraoperative data

\begin{tabular}{|c|c|c|c|}
\hline & $\begin{array}{l}\text { Auscultation group } \\
\qquad(n=34)\end{array}$ & $\begin{array}{c}\text { Sonography group } \\
(n=32)\end{array}$ & P \\
\hline \multicolumn{4}{|l|}{ Demographics } \\
\hline Age & $60 \pm 12$ & $68 \pm 10$ & 0.004 \\
\hline Sex (Male incidence) & $22(64.7 \%)$ & $17(53.4 \%)$ & 0.343 \\
\hline BMI $\left(\mathrm{kg} / \mathrm{m}^{2}\right)$ & 23.8 [21.6 to 25.7$]$ & $23.4[22.2$ to 25.7$]$ & 0.964 \\
\hline ASA & 2 [1 to 2$]$ & 2 [2 to 2] & 0.429 \\
\hline \multicolumn{3}{|l|}{ Intraoperative data } & $<0.0001$ \\
\hline Second check time (seconds) & $50 \pm 17$ & $113 \pm 29$ & $<0.0001$ \\
\hline Final check time (seconds) & $100 \pm 69$ & $131 \pm 68$ & 0.011 \\
\hline \multicolumn{4}{|l|}{ Abnormal position } \\
\hline Esophageal intubation & $3(8.8 \%)$ & $1(3.1 \%)$ & 0.614 \\
\hline Right bronchial intubation & $1(2.9 \%)$ & $1(3.1 \%)$ & 1.000 \\
\hline Too deep & $7(20.6 \%)$ & $5(15.6 \%)$ & 0.604 \\
\hline Too shallow & $5(14.7 \%)$ & $5(15.6 \%)$ & 0.918 \\
\hline Tube movement $(\mathrm{cm})$ & 1 [0 to 2] & 1 [0 to 2] & 0.684 \\
\hline Pleural adhesion & $4(17.4 \%)$ & $8(33.3 \%)$ & 0.215 \\
\hline Anesthesia time ( $\mathrm{min}$ ) & $265[180$ to 323$]$ & 265 [186 to 305$]$ & 0.847 \\
\hline Operation time (min) & $184[122$ to 220$]$ & 186 [116 to 216$]$ & 0.627 \\
\hline Crystalloid amount (ml) & 1020 [618 to 1553$]$ & 1040 [616 to 1356$]$ & 0.730 \\
\hline Transfusion (unit) & $0[0$ to 0$]$ & & 0.248 \\
\hline & & & \\
\hline
\end{tabular}

Accuracy between auscultation and sonography

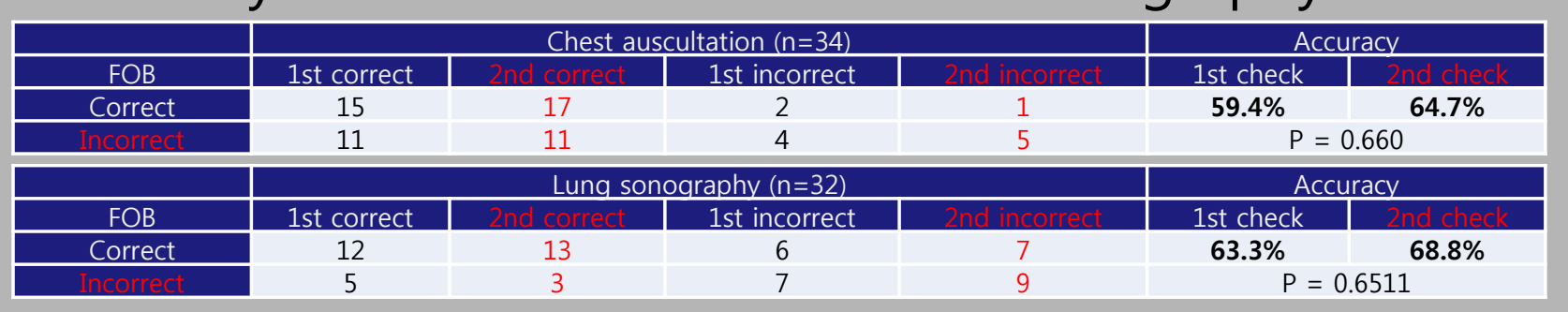

After the deletion of patient with pleural adhesion

\begin{tabular}{|c|c|c|c|c|c|}
\hline & 1st correct & 2nd dorrective & 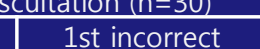 & & $\begin{array}{l}\text { Accuracy } \\
\text { 1st check } 1 \text { 2nd chec }\end{array}$ \\
\hline Correc & $\begin{array}{l}13 \\
10\end{array}$ & $\begin{array}{l}14 \\
10\end{array}$ & $\begin{array}{l}1 \\
4\end{array}$ & $\begin{array}{l}1 \\
5\end{array}$ & $60.7 \% \quad P=0.849$ \\
\hline & orrec & & 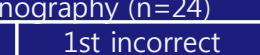 & & st ch \\
\hline & $\begin{array}{l}8 \\
4\end{array}$ & $\begin{array}{c}11 \\
1\end{array}$ & $\begin{array}{l}5 \\
6\end{array}$ & $\begin{array}{l}3 \\
9\end{array}$ & $\begin{array}{c}60.8 \% \\
P=0.088\end{array}$ \\
\hline
\end{tabular}

\section{CONCLUSION}

- Lung sonography did not show better accuracy than chest auscultation according to this preliminary data.

- Lung sonography requires more time than chest auscultation.

- Even a novice, lung sonography shows good accuracy.

- Pleural adhesion may contribute false-negative result in lung sonography. 